\title{
Efectos colaterales del COVID-19: el cuidado del adulto mayor en el hogar
}

\section{Silvana Matassini}

(iD) https://orcid.org/0000-0001-6175-3645

Instituto de Evaluación de Tecnologías en Salud e Investigación de EsSalud, Perú smatassini@gmail.com S.Matassini@2015.hull.ac.uk

RESUMEN

Este artículo busca explorar los efectos colaterales del COVID-19 con relación al cuidado de la población adulta mayor asegurada al seguro social del Perú, EsSalud, y que se encontraba confinada en sus hogares. Se realizó un estudio sobre el efecto de la pandemia en personas adultas mayores que solian asistir a un centro de adulto mayor-CAM. Se llevaron a cabo entrevistas semiestructuradas vía telefónica a los participantes. El estudio mostró que la pandemia ha ocasionado ciertas reconfiguraciones en los hogares de las personas adultas mayores, tanto en su materialidad como en las dinámicas internas.

Las personas adultas mayores - PAM que participaron en el estudio recibieron el apoyo y cuidado de sus familiares más cercanos, por lo general los que compartían el mismo hogar. Sin embargo, las debilidades en el sistema de salud podrían vulnerar a aquellas personas adultas mayores con redes de apoyo menos robustas. Los efectos colaterales de la pandemia más resaltantes en la PAM están relacionados con sus actividades recreativas, ya que han quedado paralizadas, lo que les ha ocasionado sentimientos de frustración y aburrimiento. La actual pandemia aún exige un cuidado y seguridad constante que evite poner en riesgo de contagio a la población en general, pero que también considere dentro de sus estrategias de cuidado el efecto de la salud a causa de la reducción de sociabilidad. El trabajo conjunto entre el asegurado, familias y EsSalud garantizará mejores estrategias de prevención y atención.

Palabras clave: adultas mayores, COVID-19, cuidadores familiares, redes de apoyo. 


\title{
Collateral Effects of COVID-19: Caring for the Elderly at Home
}

\author{
ABSTRACT
}

This article discusses the collateral effects of COVID-19 concerning the care of the elderly population who belong to the social security of Peru (EsSalud) and who were confined to their homes. A case study was conducted on the effect of the pandemic in older adults who used to attend an elderly care center - CAM. Semi-structured interviews were carried out via the telephone with the participants. The study showed that the pandemic caused changes in their homes and dynamics. Participants in the study are supported and cared for by their closest relatives, usually those who share the same household. However, weaknesses in the health system and social protection work could significantly harm this population group with less robust support networks. The main side effects of the pandemic in the PAM are related to their recreational activities since they have been paralyzed, causing feelings of frustration and boredom. The current pandemic still requires constant care and safety to avoid putting the general population at risk. However, it is crucial to consider the effect of the social isolation on people's health. Joint work between the insured, families and Es Salud will guarantee better prevention and care strategies.

Keywords: Older adults, COVID-19, family caregivers, support networks. 


\section{INTRODUCCIÓN}

A finales de 2019, se identificó en China un nuevo coronavirus al que se denominó COVID-19. Debido a su velocidad de expansión y gravedad, el 11 de marzo de 2020 la Organización Mundial de la Salud - OMS lo declaró como pandemia (OMS, 2020). Ante esta crisis de salud mundial, se han implementado globalmente medidas de salud pública para la mitigación de la pandemia, tales como el confinamiento obligatorio y el distanciamiento social, lo cual incluyó: restricción de viajes, medidas en el entorno escolar, laboral y comunitario, entre otros (Martín et al., 2020).

La aplicación de estas medidas tuvo como objetivo retrasar el momento de máxima incidencia y reducir el número total de casos de infectados. Sin embargo, los efectos colaterales de esta pandemia están relacionados, por un lado, con las medidas de distanciamiento social y confinamiento que ponen en peligro, restringen y, en algunos casos, anulan ciertos tipos de sociabilidad de las poblaciones más vulnerables.

El confinamiento ha producido cambios en las dinámicas familiares que han implicado negociaciones y adaptaciones internas. Se han presentado modos de violencia en aquellos hogares donde no existían antes de la crisis sanitaria, y se han incrementado los episodios de violencia en familias donde anteriormente ya se presentaban estas situaciones. Los tipos de violencia que se han incrementado en tiempos de pandemia son violencia física, psicológica, sexual y económica dirigida a niños, adolescentes, mujeres y adulto mayor (Medina et al., 2020; Villalba, 2021). Con relación a la población adulta mayor, las ONU advierte sobre un probable incremento de casos de violencia y maltrato hacia esa población (Naciones Unidas, 2020). En el Perú, aún se tiene pendiente una mayor indagación al respecto. No obstante, durante los meses de marzo y octubre de 2020 el Centro de Emergencia Mujer indicó una caída nacional en las llamadas usuales denunciando casos de violencia, lo que llevaría a pensar que esto podría estar vinculado con las dificultades para reportar estas situaciones de manera telefónica 
o virtual, la convivencia con agresores o mayor carga en tareas domésticas por las posibles víctimas, incluyendo las personas adultas mayores (Bravo et al., 2021).

Esta situación, conjuntamente con la expansión de la enfermedad, podría colocar a las personas que no cuentan con redes sociales de apoyo, o se encuentran alejadas de espacios públicos y con poco acceso a servicios de salud en posiciones vulnerables. Durante la primera etapa de la pandemia, las noticias en diversos medios de comunicación resaltaban las muertes de PAM en total aislamiento, abandono, sin poder ser asistidas a tiempo (OPS/OMS / Organización Panamericana de la Salud, 2020).

Este artículo presenta los resultados de un estudio que buscó explorar los efectos colaterales del COVID-19 con relación al cuidado de la población adulta mayor asegurada al seguro social del Perú, EsSalud, y que se encontraba confinada en sus hogares.

El cuidado, como sugieren diversos autores, es un concepto que va más allá del hecho de dar y recibir: está relacionado con aspectos culturales, con lógicas y normativas situadas (Aulino, 2016; Sánchez-García et al., 2017). Además, el cuidado abarca no solo las relaciones sociales, sino también aspectos materiales y económicos, culturales, históricos y biológicos. Estos elementos están indisolublemente ligados entre sí, lo que significa que analizar el cuidado debe significar también analizar estas múltiples formas de relación. Pero el cuidado también es dinámico, es decir, las relaciones que se involucran en los procesos de cuidado varían a lo largo del tiempo, no son estáticas (Aulino, 2016; Sánchez-García et al., 2017; Seaman et al., 2019).

Se realizó un estudio sobre el efecto de la pandemia en personas adultas mayores que solían asistir a un centro del adulto mayor - CAM. Los CAM tienen como objetivo principal mejorar la calidad de vida de las personas adultas mayores, proporcionando espacios para el desarrollo de programas de integración familiar, intergeneracional, socioculturales y recreativos. Entre los servicios que proporcionan, se encuentran: talleres de educación emocional, de memoria, de autocuidado, de arte, de cultura física, de turismo social de huertos y de crianza de animales menores, así como comedores sociales y eventos deportivos y recreativos. Los CAM, además, son espacios en donde se despliegan apoyos emocionales y de cuidado entre las personas adultas mayores, debido a los cambios en las circunstancias de la vida que pueden experimentarse durante la vejez. Por ejemplo: la viudez, reconfiguraciones en el hogar como consecuencia de la mudanza de los hijos, desafíos relacionados con temas de salud, etc., que pueden afectar su identidad y sentido de pertenencia (Chambers, 2000; Moglen, 2008; Quadagno, 2017). 
Desde la pandemia, los CAM han suspendido sus actividades presenciales, realizando controles y monitoreos de los participantes a través de llamadas telefónicas y videollamadas. Sin embargo, la adaptación a estos nuevos formatos no se da de manera homogénea, lo que ocasiona que muchas personas adultas mayores se desvinculen de estos espacios, reduciendo así sus alternativas de sociabilidad.

\section{METODOLOGÍA}

El presente estudio buscó explorar los efectos colaterales del COVID-19 en relación con el cuidado de la población adulta mayor asegurada al seguro social del Perú que asistían a un CAM en la ciudad de Lima. Para ello, se abordaron los aspectos socioeconómicos de las personas adultas mayores, la composición de sus hogares, el efecto de la pandemia en su salud, sus redes de cuidado y, los principales cambios personales y familiares a partir de la pandemia. El propósito fue comprender el cuidado durante la actual crisis sanitaria actual en diálogo con las estructuras y capitales acumulados en las trayectorias de estas personas.

El diseño del estudio fue cualitativo narrativo, donde se emplearon entrevistas semiestructuradas a los participantes.

En los diseños narrativos el investigador recolecta datos sobre las experiencias de las personas y su entorno. Se pueden analizar diversos aspectos: la historia de vida, un fenómeno en particular, el ambiente (tiempo y lugar) en el cual vivió la persona o grupo, o sucedieron los hechos; las interacciones (Salgado Lévano, 2007). De esta información proporcionada, el investigador encontrará categorías que ayuden a responder los principales cuestionamientos que conducen el estudio.

En el estudio, participaron personas adultas mayores que asistieron a un CAM de la ciudad de Lima, y sus cuidadores. El recojo de información se llevó a cabo aplicando entrevistas semiestructuradas vía llamada telefónica. Los participantes fueron contactados previamente por un miembro del personal de salud del CAM. En una primera llamada, el personal de salud informó a los usuarios sobre los objetivos del estudio y se les preguntó si deseaban formar parte de él. Con las personas que aceptaron participar en la investigación se coordinaron fechas y horas posibles para realizar la segunda llamada y concretar las entrevistas.

Las citas textuales de los participantes, compartidas en el estudio, se presentan con nombres ficticios, respetando la confidencialidad de las personas adultas mayores. 
La muestra fue intencional. Personal de salud del CAM proporcionó una lista de personas adultas mayores que, según consideraba, podría participar del estudio dada su mayor asistencia al centro antes de la pandemia. De esta lista se pudo entrevistar a tres mujeres y dos varones entre 64 y 78 años de edad. A continuación, se presenta una síntesis de sus vidas.

Liliana. Al momento del estudio, y desde antes de la pandemia, vivía con sus hijas e hijos. Tiene 74 años. En relación con su salud, la señora sufre de asma; sin embargo, expresa que no le representa una molestia y que la tiene controlada. Liliana no concluyó sus estudios técnicos y ha realizado por muchos años trabajos informales para apoyar económicamente a su hogar. Su familia es el principal soporte de cuidado, físico, emocional y entre todos contribuyen económicamente al hogar.

María. Al momento del estudio y desde antes de la pandemia, vivía con su esposo, su hija y dos nietos. Aunque sufre de hipertensión y parte de su rutina diaria es tomar pastillas para controlar la enfermedad, manifiesta sentirse saludable e independiente. Tiene 67 años y ha realizado por muchos años trabajos informales para apoyar económicamente a su hogar.

Carlos. Al momento del estudio y desde la pandemia vive con sus hijos, quien se mudaron con él a su vivienda para apoyarse y cuidarse mutuamente. El señor Carlos tiene 78 años y su principal actividad económica desde antes de jubilarse hasta la actualidad ha estado relacionada con el transporte. Hasta antes de la pandemia, aparte de su jubilación, el señor Carlos recibía ingresos adicionales por actividades vinculadas con transporte que llevaba a cabo esporádicamente.

Iván. El señor Iván tiene 72 años. Enviudó en 2016 y desde antes de la pandemia vive con dos de sus tres hijos. El hijo mayor vive en el extranjero y no lo ve desde hace trece años. Es el único participante profesional y cuenta que se ha desempeñado principalmente como magistrado.

Clara. Al momento del estudio, y desde antes de la pandemia, Clara vive con sus hijas. Sin embargo, con la llegada de la pandemia decidieron mudarse a un lugar más amplio para poder acoger a sus sobrinos y apoyarse mutuamente ante la crisis e incertidumbre que se avecinaba. Ella es viuda y recibe la pensión de quien fuera su esposo. Eso, junto con los ingresos de sus hijas y sobrinos, contribuye a la economía del hogar.

Se utilizó la siguiente guía de entrevistas para el abordaje del estudio: 


\begin{tabular}{cc}
\hline Temas & Contenido \\
\hline $\begin{array}{c}\text { Realidad } \\
\text { socioeconómica }\end{array}$ & $\begin{array}{c}\text { Actividades económicas } \\
\text { Vivienda } \\
\text { Servicios básicos }\end{array}$ \\
\hline Educación & Nivel de educación alcanzado \\
Intereses
\end{tabular}

Estos ejes permitieron desplegar largas conversaciones y profundizar en la experiencia de las personas adultas mayores a partir del confinamiento. La investigación pretende contribuir a visibilizar los desafíos físicos y emocionales que afectan a la población adulta mayor y que son parte importante de sus cuidados durante la crisis sanitaria actual. Asimismo, busca discutir sobre la red de cuidados alrededor de las personas adultas mayores, los agentes involucrados y la necesidad de reforzar aquellos aspectos que impidan o reduzcan una vida digna para este grupo poblacional. 


\section{RESULTADOS}

En esta sección se presentan los resultados del estudio, empezando por los cuidados de las PAM con relación a la familia y a los nuevos formatos de atención virtual por EsSalud. Posteriormente se discutirán los hallazgos referidos al efecto de la pandemia en la sociabilidad y la salud mental de los participantes. A continuación, se tratará sobre aquellos aspectos relacionados con las reconfiguraciones domésticas a partir de la crisis sanitaria mundial y las principales redes de apoyo de las PAM. Finalmente, se culminará esta sección con los resultados acerca del impacto económico como producto de la pandemia, percibidos por los participantes.

\subsection{Cuidados familiares y la virtualidad de la salud}

El cuidado se analiza en dos dimensiones. Por un lado, los cuidados que se reciben del sistema de salud y, por otro, el cuidado cotidiano, como la alimentación, aseo y condiciones de vida en general. Las personas adultas mayores que participaron en el estudio se encuentran en situación de independencia física y no padecen de enfermedades que impidan su movilidad ni sus actividades cotidianas principales. Además, cuentan con redes familiares cercanas, con vínculos fuertes y relaciones armoniosas. Por ello, el cuidado y el apoyo recíproco, entre la familia y el adulto mayor y viceversa, fue resaltante y se centró principalmente en los aspectos emocionales y logísticos (recojo de medicinas por los miembros familiares más jóvenes, cuidado de menores de edad por las PAM y colaboración en tareas domésticas entre los miembros del hogar).

Por ahora no necesito nada, antes me han hospitalizado y mis hijos me llevaban a las terapias, pero ya estoy bien y yo ayudo en la casa, todos ayudamos, porque ahora estamos todos metidos. Hay que ser solidarios, da mucho miedo esta enfermedad, pero hay que estar unidos para seguir con salud (señor Iván).

Sin embargo, en los primeros momentos de la pandemia, todas las entidades sanitarias estaban centradas en las atenciones por el COVID-19. Las personas adultas mayores entrevistadas experimentaron cambios significativos en sus atenciones médicas debido al congestionamiento y colapso de los diversos canales de comunicación con el seguro social, la reducción de algunas especialidades de salud y el traslado de citas a la modalidad virtual. La adaptación de estos nuevos formatos son experiencias individuales. Asumir el uso y familiaridad de ellos 
en toda la población puede anular la necesidad de educar sobre el traslado a estas tecnologías y aumentar las brechas en el acceso a servicios de salud. Por ejemplo, el señor Carlos mencionó no tener ningún problema con la modalidad virtual: se siente muy cómodo durante sus atenciones con su médico de familia y manifestó que los nuevos canales de atención virtual no han resultado una barrera para el monitoreo de su salud. No obstante, la señora María afirma que, aunque se siente una persona bastante independiente, el uso de la tecnología no le es muy amigable y se siente más confiada cuando puede asistir y ser atendida en consultorios médicos.

El servicio de tecnologías de información y comunicación en salud —comúnmente conocido como telesalud - busca acercar los servicios de salud a la población con diversas dificultades de acceso en general. Sin embargo, desde el inicio de la pandemia, este servicio busca principalmente continuar con las atenciones a la población asegurada. El uso de tecnologías - ya sea una videollamada a través del celular o computadora - aún no es un servicio consumido fácilmente por toda la población, ya sea por su posibilidad de acceso o por conocimiento de uso. Las tecnologías si bien pueden contribuir a una mayor integración de la población también pueden estar perpetuando brechas. Según reportes de la Comisión Económica para América Latina y el Caribe - Cepal, aún prevalecen marcadas diferencias en el acceso y uso de tecnologías de comunicación por la población adulta mayor. Aun cuando el acceso y el uso de la internet ha aumentado en todos los países de la región entre la población de sesenta años a más, los niveles de uso son bajos comparados con los otros grupos etarios. Por lo tanto, si bien muchas PAM en la región viven en hogares con acceso a internet, un porcentaje importante aún no se familiariza con su uso. Las PAM son el sector con menor acceso y capacidades de uso del internet ( $26,9 \%$ ) y TIC ( $78 \%$ cuenta con celular), lo que evidencia la existencia de una brecha digital generacional (INEI, 2021).

\subsection{Confinamiento y salud mental}

Además de los aspectos relacionados directamente con el colapso y reconfiguración de los servicios sanitarios al momento inicial de la pandemia, la incertidumbre de la situación y el ser testigos diarios de mayores casos de contagios y muertes afectó la tranquilidad familiar. Esto se reflejó en dificultades para conciliar el sueño, desórdenes alimenticios o miedo constante cuando un miembro del hogar salía de casa. El efecto de la pandemia en la salud mental de la población en general se incrementó, además de por la incertidumbre del mundo sobre diversos 
aspectos de la enfermedad, por la desinformación en las redes sociales y medios de comunicación, a menudo impulsado por noticias erróneas y por la mala comprensión de los mensajes vinculados con el cuidado de la salud (AleixandreBenavent et al., 2020). Tal como compartió la señora Liliana: «Antes éramos más unidos; ahora no podemos ir a comer con la familia, estamos separados por temor, a veces ni hambre me daba», o la señora Clara, quien mencionó parte de sus preocupaciones diarias desde la pandemia: «Mi hija y mis sobrinos trabajan fuera, mi mente no descansa hasta que regresan, pero igual tengo miedo de que se enfermen y se debiliten, no para, siempre hay miedo».

El temor al contagio y a la muerte anularon dinámicas de socialización familiar más amplia. Liliana y Clara aprendieron a vivir con un miedo permanente expresado en insomnios o dolores de cabeza. Ante este escenario, su principal respaldo fue la familia con la que compartían la misma vivienda. Para la mayoría de participantes, los momentos de risas con sus familiares, las conversaciones prolongadas y la compañía en general contribuyeron a navegar los peores momentos de la pandemia. Para el señor Iván, sin solidaridad familiar la pandemia hubiese afectado emocionalmente a su hogar. O, como comentó el señor Carlos con respecto a las relaciones familiares desde la pandemia, «somos apoyo casi inmediato, porque por la misma circunstancia que vivimos, tenemos que ver por la familia. Eso lo estamos haciendo para salir adelante».

Las medidas de confinamiento y el temor a la misma enfermedad han suspendido el relacionamiento de las personas adultas mayores con otros pares. La señora Clara nos cuenta que ahora sus actividades se centran al hogar, a cuidarse y estar atenta a las noticias. Menciona que el miedo ha hecho que elimine de su mente cualquier deseo o proyecto futuro. Está muy asustada y extraña su vida participando de las actividades del CAM.

Ya a mi edad, ya no deseo, ya no proyecto nada. Igual pues hago todos los quehaceres de la casa. Lo que más me gusta es el tejido y participar en talleres del CAM, me gusta viajar, los paseos los extraño.

Del mismo modo, la señora Liliana comenta que antes ella iba al CAM y «era feliz», se divertía, se olvidaba de sus problemas. Desde la pandemia ella dice no tener la misma motivación para hacer cosas: está pensando constantemente en que ella ni su familia se contagien.

Ahora mi interés es cuidarme, a estas alturas de la vida y de la pandemia, tal como está sucediendo. No pienso ya en otra cosa como antes. No sé si podremos ir al CAM otra vez, y es triste pues, pero así es. 
El señor Iván menciona que extraña salir a la calle con tranquilidad, conversar con la gente sin miedo. Se siente protegido en su hogar, con su familia, pero admite sentirse por momentos frustrado y aburrido por la incertidumbre de no saber cuándo volverá «la vida normal».

El efecto de estas restricciones en el marco de la crisis de salud global — como demuestra la literatura - puede ocasionar problemas en la salud mental de la población (Sureims et al., 2021). La incertidumbre del avance de la pandemia y la desinformación, conjuntamente con menores espacios de distracción, pueden colocar a las personas adultas mayores en situaciones de riesgo, acrecentando incluso posibles comorbilidades (Caycho-Rodríguez et al., 2021; Orozco-Rocha y González-González, 2021; Pinazo-Hernandis, 2020). Además del mayor riesgo al contagio de la población adulta mayor debido a un sistema inmune probablemente más debilitado que en edades tempranas. La soledad y el aislamiento social son determinantes importantes en su salud e influyen decisivamente en su bienestar y calidad de vida. El confinamiento y el aislamiento social aumentan el problema preexistente de soledad y las consecuencias en la salud de la desconexión social que existían ya desde antes de la pandemia (Callís-Fernández et al., 2021).

De acuerdo con la literatura, la soledad se puede experimentar por diferentes causas. Por un lado, es un sentimiento subjetivo negativo, extraño, incorrecto, feo, o incluso vergonzoso. En general, la soledad no está bien: es un tabú, pues es desagradable y angustiante (Burton-Shepherd, 2015; Dahlberg y McKee, 2014; Victor et al., 2005). El que se siente solo es de «rango inferior» y tiene un «estatus inferior» que el que no lo está (Dahlberg y McKee, 2014). Pero este sentimiento negativo de soledad también se experimenta a causa de aislamientos físicos, está relacionado con la distancia y la accesibilidad, también tiene un enfoque geográfico (Burton-Shepherd, 2015). Asimismo, el número de contactos con los que se cuente y la frecuencia interacciones con ellos mismos puede determinar la experiencia de soledad y aislamiento de las personas (Price, 2015).

La conexión entre soledad, aislamiento y salud en la vejez se ha estudiado extensamente entre diferentes disciplinas (Dickens et al., 2011; Price, 2015). Estos estudios identifican que la soledad y el aislamiento podrían representar una amenaza para las personas mayores, afectando su salud física y mental. Por ejemplo, como argumenta Price (2015), la soledad está asociada con diferentes tipos de morbilidades y tiene una conexión significativa con la depresión y el suicidio. La presión arterial, la mala salud física y la mala alimentación también están relacionadas con estas afecciones (Cacioppo et al., 2011). Kharicha et al. (2007) afirmaron que el riesgo para la salud asociado con el aislamiento y la soledad en los adultos mayores es similar al del tabaquismo. En el trabajo de 
Learner (2011), la soledad se presenta como un problema de salud pública importante debido a los efectos que puede causar en la salud de las personas: depresión, alcoholismo, mala alimentación y falta de ejercicio. También puede desencadenar hipertensión arterial, afectar los sistemas inmunológico y cardiovascular y se ha relacionado con la aparición de la enfermedad de Alzheimer.

Ante este panorama, tanto en el Perú como en otras partes del mundo, las atenciones remotas o virtuales han buscado ofrecer atenciones médicas como programas de recreación y aprendizaje para este grupo poblacional, convirtiendo a las TIC en una herramienta fundamental para el aprendizaje, capacitación y refuerzo de relaciones familiares, intergeneracionales e interpersonales. Sin embargo, como hemos señalado, aún mantiene el riesgo de excluir a un importante grupo de la población (Albañil-Delgado et al., 2020; Concepción-Breton et al., 2020).

Las actividades de socialización externa provista por los CAM son las que más añoran las personas adultas mayores. Es el espacio doméstico y la familia el nuevo canal para ese despliegue de sociabilidad. Sin embargo, dada la yuxtaposición de diversas prácticas sociales dentro de un mismo espacio, el despliegue de actividades recreacionales dentro del hogar por las personas adultas mayores es algo que no se visibilizó en las entrevistas, quizá porque ninguno de los participantes realizaba alguna actividad virtual dentro del CAM o en ningún otro espacio.

\subsection{Configuraciones domésticas y redes de apoyo}

Las familias del grupo de entrevistados, al momento del estudio, mostraban características diversas en cuanto a su composición: por un lado, las formadas por solo dos miembros y por otro, familias extendidas de hasta nueve personas. Ninguno de los participantes describió las relaciones entre los miembros de su hogar como negativas, más bien son calificadas de perfectas, excelentes, «todos bien unidos, todos bien comunicativos» (señora Clara).

El efecto de la pandemia obligó a muchas familias a un cambio en las dinámicas e infraestructuras domésticas. Muchos de los procesos de socialización de las personas se dieron, a causa del confinamiento, dentro de sus hogares, lo que obligó a transformar dichos espacios en oficinas, aulas virtuales, consultorios médicos, áreas recreativas, etc. Es decir, la vivienda se convirtió en el encuentro de actividades usualmente desplegadas externamente con las tareas típicamente domésticas, como el cuidado, la alimentación, el ocio, etc. Al mismo tiempo, el temor a la socialización externa al hogar ante la posibilidad del contagio incrementó la ansiedad, frustración y miedo en la población (Hernández et al., 2020). 
Las personas adultas mayores entrevistadas se caracterizaron por vivir en viviendas propias o de propiedad familiar. Al iniciarse la pandemia, la necesidad de cuidado y apoyo mutuo fue fundamental para decidir nuevas configuraciones del hogar. Por ejemplo, la familia de la señora Clara decidió mudarse a una vivienda más amplia para poder invitar a dos sobrinos a vivir con ellos. O el caso del señor Carlos, donde su hija y sus dos hijos se mudaron a su vivienda para poder cuidarse y apoyarse mutuamente, o la señora María, quien junto a su esposo apoyan a su hija en el cuidado de sus dos nietos para que ella pudiera trabajar.

Las personas entrevistadas aseguraron que las funciones en su hogar son compartidas. Esto se vincula con que todos los participantes coincidieron en que no cuentan con miembros dependientes, que requieran cuidados especiales o, en palabras de la señora María, «yo y mi esposo todavía no necesitamos cuidado, nos valemos por nosotros mismos muy bien».

Fuera del hogar, tal como mencionan los participantes, normalmente son la familia y algunos de los vecinos la red de apoyo más cercana con la que solían contar. Sin embargo, desde el inicio de la pandemia, el miedo y el temor a la socialización por un posible contagio ha interrumpido el flujo de comunicación y de soporte. No obstante, se han generado estrategias, donde es la información el bien más útil y valorado. Las conversaciones grupales por WhatsApp entre vecinos, que usualmente eran canales de comunicación creados, principalmente, para articular coordinaciones orientadas a prevenir el delito, son ahora espacios para compartir información sobre los diversos aspectos relacionados con la pandemia: acceso a medicamentos, oxígeno, camas en hospitales, etc. Aunque se reconocen limitaciones en el uso de estas tecnologías de comunicación por los adultos mayores, la mayoría los entrevistados están familiarizados con el uso de redes sociales como WhatsApp para la comunicación, principalmente, con miembros de su familia extensa.

Las personas adultas mayores y sus familiares tuvieron que enfrentar cambios abruptos en su cotidianeidad a raíz de la pandemia. Kessler (2009) utiliza el concepto de «distanciamiento del habitus» para discutir situaciones en las cuales se deben generar estrategias de adaptación y enfrentamiento ante situaciones de pobreza. Así pues, en el caso de las personas adultas mayores del estudio, el cambio violento que supuso la pandemia condujo a un desorden en las rutinas de sus hogares, ocasionando un «distanciamiento del habitus». Esta distancia del habitus obligó a que «reinventen lo cotidiano». En este sentido, el desafío no solo fue ni es adaptarse al confinamiento sino a la necesidad de restituir un orden externo al interior del hogar (Kessler, 2009). 


\subsection{Efectos económicos de la pandemia}

De las cinco personas entrevistadas, solo una de ellas —el señor Iván— - se jubiló como profesional, luego de realizar labores de magistrado. El señor Carlos trabajó en una empresa de transportes. La señora Clara recibe la pensión de su difunto esposo desde hace más de siete años. Además, menciona no haber realizado actividades laborales que le hayan permitido recibir una pensión. Las señoras María y Liliana se dedicaban a trabajos de limpieza en trabajos informales. La inequidad de género es un aspecto que sobresale principalmente en relación al acceso a trabajo y educación. Se ha argumentado que las vidas de las mujeres son impactadas por cuestiones de género (Giuliano, 2017) debido a las expectativas sociales sobre sus roles. Por ejemplo, las actividades domésticas, responsabilidades del cuidado familiar, menor acceso a educación y oportunidades laborales. El estudio de Ramos y Tirado (2019), sobre trayectorias laborales de personas adultas mayores en Lima, encontró como parte de sus hallazgos, mayores situaciones de vulnerabilidad en mujeres, dado que el acceso y desempeño en el mercado laboral —así como su fondo de pensiones - fueron negativamente afectados por las responsabilidades domésticas asumidas y esperadas.

Los resultados del estudio muestran que las personas adultas mayores y sus familiares no han percibido un impacto económico negativo significativo en sus hogares, ya sea por sus ingresos como pensionistas o familiares de pensionistas, soporte material y económico familiar y actividades informales o «cachuelos». Sin embargo, han ocurrido reducciones de ingresos e inestabilidad de empleos. Uno de los participantes mencionó que a raíz de la pandemia tuvo que dejar de realizar «cachuelos» que complementaban sus ingresos por jubilación.

Antes de la pandemia, yo a dos personas les manejo su carro, hacemos cosas de esas personas, entonces no es todos los días, pero de vez en cuando, a veces dos veces a la semana, $\mathrm{o}$ tres, a veces toda la semana, como a veces no hay nada. Y después a veces voy con algunos señores que los ayudo a cobrar por ejemplo su jubilación, a hacer cosas, pagar el teléfono, todo eso los ayudo a esos señores, que ya tienen 86 hasta 90 años (señor Carlos).

Las personas que participaron en el estudio forman parte de redes familiares de apoyo y cuidado y en ese sentido, el apoyo material y económico se ha acentuado para afrontar los diferentes desafíos a causa de la pandemia. Estudios en la región señalan que los aspectos que influyen en la vulnerabilidad económica de las personas adultas mayores son: menores ingresos personales y familiares, edad avanzada, personas con menos escolaridad, hogares unipersonales, poca o escasa 
ayuda de hijos y familiares cercanos (González-González et al., 2021; Ramos Bonilla y Tirado Ratto, 2019). Por lo tanto, la acumulación de recursos económicos está relacionada también con el empleo de las personas adultas mayores en la actualidad o en los años laborales, al apoyo familiar, al capital cultural y educativo que acumuló y a sus beneficios. En este estudio, las redes familiares de apoyo contribuyen emocional, material y económicamente al cuidado de las PAM. Además, se puede observar que el apoyo familiar también contaba con la participación de las personas adultas mayores, como por ejemplo en el cuidado de los nietos y el acceso a la vivienda. Así, no es meramente un rol de receptor el que asumen las personas adultas mayores dentro de sus hogares. Por ejemplo, hay estudios que han demostrado que aquellas familias que realizan trabajos fuera de casa durante la pandemia, han organizado el cuidado de los hijos e hijas alrededor de abuelos y abuelas (Serrano-Martínez, 2020).

Mi hija se mudó a casa apenas empezó la pandemia y así podemos ayudarla cuidado a mis nietos, viendo que hagan sus tareas y que la dejen trabajar, porque todos en casa veces es un poco complicado. Pero nos organizamos, mi esposo limpia un poco mientras yo veo a los chicos y así la vamos pasando (señora María).

Hay aspectos importantes que contribuyen de manera indirecta con la economía familiar en los hogares con personas adultas mayores y que son claves para la lucha contra la pobreza, pero que son poco señalados en la literatura porque no representan un aporte monetario directo (Ramos, 2009).

\section{CONCLUSIONES}

Las medidas de mitigación ante la actual crisis de salud global han incluido al confinamiento como una de las principales estrategias de lucha contra el contagio del COVID-19. Esta experiencia obliga a adaptaciones abruptas e irrumpe en los diferentes aspectos de la vida de las personas. El espacio doméstico se convierte en el lugar en donde confluyen diversos tipos de actividades y emociones, a la vez que se experimentan convivencias nuevas y prolongadas entre las familias.

Dadas las características socioeconómicas de los participantes y su independencia física y económica, los cuidados durante la pandemia se han centrado en el apoyo emocional y logístico de sus familiares más cercanos. Sin embargo, en América Latina más de ocho millones de adultos mayores viven en situación de dependencia funcional. Es decir, estas personas no pueden llevar a cabo de 
manera independiente al menos una actividad básica de la vida diaria: bañarse, comer, ir a los servicios higiénicos, vestirse, caminar, o acostarse y levantarse de la cama. En la región, la dependencia funcional afecta al 12\% de las personas de sesenta años a más, y a casi el $27 \%$ de los mayores de ochenta años (Cafagna et al., 2019). En esta parte del globo, el apoyo informal no remunerado - ya sea a través de la ayuda de familiares o amigos- ha sido la principal fuente de apoyo a esta población, siendo las mujeres de la familia las que principalmente asumen este rol (Cafagna et al., 2019). Esta situación se acentúa ante la crisis de salud actual, ya que se debe lidiar con el difícil balance entre trabajo y cuidados, posibles hacinamientos domésticos, inestabilidad económica, reducción de servicios y atenciones de salud, el síndrome de Burnout (ansiedad, agotamiento, temor y miedo de no cumplir de forma adecuada con las labores de asistencia), etc. (Martínez-López et al., 2021). Por lo tanto, buscar mejoras en la atención de las personas adultas mayores también requerirá de la mirada al cuidador, sus necesidades y efectos personales y profesionales, las implicancias para mujeres y hombres y la urgencia de presentar respuestas desde el Estado.

Las debilidades en el sistema de salud y en trabajos de protección social podrían vulnerar especialmente a este grupo poblacional con redes de apoyo menos robustas y menor capital económico. Por ende, es crucial estar preparados y prever futuras crisis sanitarias que los pongan en riesgo. Resulta fundamental generar evidencia de calidad sobre sus impactos en la población mayor y las posibles intervenciones para prevenirlos, revertirlos o paliarlos. En ese sentido, el trabajo conjunto entre el asegurado, familias y entidad de salud garantizará mejores estrategias de prevención y atención.

\section{REFERENCIAS}

Albañil-Delgado, S., Machain, P. A. y Guerrero-Castañeda, R. F. (2020). Técnicas de terapia com arte no domicílio como cuidados de enfermagem a idosos em contingência devido ao COVID-19. Cogitare Enfermagem, 25(0), Article 0. https://doi. org/10.5380/ce.v25i0.73883

Aleixandre-Benavent, R., Castelló-Cogollos, L. y Valderrama-Zurián, J.-C. (2020). Información y comunicación durante los primeros meses de COVID-19. Infodemia, desinformación y papel de los profesionales de la información. El Profesional de la Información (EPI), 29(4). https://doi.org/10.3145/epi.2020.jul.08

Aulino, F. (2016). Rituals of care for the elderly in northern Thailand: Merit, morality, and the everyday of long-term care. American Ethnologist, 43, 91-102. https:// doi.org/10.1111/amet.12265 
Bravo, V. B. R., Hilario, J. D. S., Ojeda, V. P. C. y Torres, C. N. B. (2021). Vivencias y sentido de vida del adulto mayor víctima de violencia familiar en tiempos de Covid 19. Universidad y Sociedad, 13(4), 499-504.

Burton-Shepherd, A. (2015). Face in the crowd: Loneliness and isolation in older people. https://doi.org/10.12968/NREC.2015.17.12.698

Cacioppo, J. T., Hawkley, L. C., Norman, G. J. y Berntson, G. G. (2011). Social isolation. Annals of the New York Academy of Sciences, 1231(1), 17-22. https://doi. org/10.1111/j.1749-6632.2011.06028.x

Cafagna, G., Aranco, N., Ibarrarán, P., Medellín, N., Oliveri, M. L. y Stampini, M. (2019). Envejecer con cuidado: Atención a la dependencia en América Latina y el Caribe. Inter-American Development Bank. https://doi.org/10.18235/0001972

Callís-Fernández, S., Guarton-Ortiz, O. M., Cruz-Sánchez, V. y de-Armas-Ferrera, A. M. (2021). Estados afectivos emocionales en adultos mayores solos en aislamiento social durante la pandemia COVID-19. Revista Electrónica Dr. Zoilo E. Marinello Vidaurreta, 46(1), Article 1. http://revzoilomarinello.sld.cu/index. php/zmv/article/view/2491

Caycho-Rodríguez, T., Barboza-Palomino, M., Ventura-León, J. y Cabrera-Orosco, I. (2021). La voluntad de vivir en tiempos de la COVID-19: Un estudio con adultos mayores peruanos. Revista Española de Geriatria y Gerontologia, 56(1), 56-57. https://doi.org/10.1016/j.regg.2020.09.007

Chambers, P. (2000). Widowhood in later life. En Women Ageing. Routledge.

Concepción-Breton, A., Corrales-Camacho, I., Córdoba, M. E., Acosta-Hernández, M. E., Larancuent-Cueto, O. I. y Cruz-Morel, Y. L. D. L. (2020). Sondeo de casos en personas mayores sobre actividades cotidianas y utilización de tecnologías de la información y la comunicación (TIC) en tiempos de pandemia. Revista Tecnológica-Educativa Docentes 2.0, 9(2), 132-150. https://doi.org/10.37843/ rted.v9i2.156

Cronología de la respuesta de la OMS a la COVID-19 (s. f.). Recuperado 16 de julio de 2021, de https:/www.who.int/es/news/item/29-06-2020-covidtimeline

Dahlberg, L. y McKee, K. J. (2014). Correlates of social and emotional loneliness in older people: Evidence from an English community study. Aging \& Mental Health, 18(4), 504-514. https://doi.org/10.1080/13607863.2013.856863

Dickens, A. P., Richards, S. H., Greaves, C. J. y Campbell, J. L. (2011). Interventions targeting social isolation in older people: A systematic review. BMC Public Health, 11(1), 647. https://doi.org/10.1186/1471-2458-11-647

Giuliano, P. (2017). Gender: An Historical Perspective (№ w23635). National Bureau of Economic Research. https://doi.org/10.3386/w23635 
González-González, C. A., Orozco-Rocha, K., Samper-Ternent, R. y Wong-Luna, R. (2021). Adultos mayores en riesgo de COVID-19 y sus vulnerabilidades socioeconómicas y familiares: un análisis con el ENASEM. Papeles de Población, 27(107), 141-165.

Hernández, D. V., Toledo, Y. I. y Lazo, R. V. (s. f.). Consejo de Redacción de Convivencia, 60 .

INEI (s. f.). Recuperado 14 de octubre de 2021, de https://www.inei.gob.pe/media/ MenuRecursivo/boletines/boletin_adulto_mayor_1.pdf

Kessler, G. (2009). Redefinición del mundo social en tiempos de cambio: Una tipología para la experiencia de empobrecimiento. Desde abajo: la transformación de las identidades sociales. Buenos Aires: Biblos. Recuperado el 26 de agosto de 2021, de http://www.memoria.fahce.unlp.edu.ar/library? $\mathrm{a}=\mathrm{d} \& \mathrm{c}=$ libros \&d=Jpm4048

Kharicha, K., Iliffe, S., Harari, D., Swift, C., Gillmann, G. y Stuck, A. E. (2007). Health risk appraisal in older people 1: Are older people living alone an «at-risk» group? The British Journal of General Practice: The Journal of the Royal College of General Practitioners, 57(537), 271-276.

Las personas mayores de 60 años han sido las más afectadas por la COVID-19 en las Américas-OPS/OMS |Organización Panamericana de la Salud.(s.f.). Recuperado 31 de agosto de 2021, de https://www.paho.org/es/noticias/30-9-2020-personasmayores-60-anos-han-sido-mas-afectadas-por-covid-19-americas

Learner, S. (s. f.). Sick of feeling lonely. https://doi.org/10.7748/ns.26.2.18.s24

Martín, L. V., Rodríguez, M. F. y DurÃ, S. C. (s. f.). Medidas frente a la pandemia por coronavirus (COVID-19). 13.

Martínez-López, J. Á., Lázaro-Pérez, C., Gómez Galán, J. y Raducea, M. (2021). Aproximación a los cuidados de larga duración durante el pico de la COVID-19 en la Región de Murcia. http://riberdis.cedd.net/handle/11181/6318

Medina, D. C. M., Guayacundo, M. O. B., Encalada, L. M. A. y Estupiñán, A. M. M. (2020). Violencia intrafamiliar en el marco de la emergencia sanitaria por el COVID-19. CienciAmérica: Revista de divulgación científica de la Universidad Tecnológica Indoamérica, 9(Extra 2), 261-267. https://doi.org/10.33210/ ca.v9i2.316

Moglen, H. (2008). Ageing and transageing: Transgenerational hauntings of the self. Studies in Gender and Sexuality, 9(4), 297-311. https://doi. org/10.1080/15240650802370668

Naciones Unidas. (s. f.). Los efectos de la COVID-19 en las personas de edad (p. 20). 
Orozco-Rocha, K. y González-González, C. (2021). Vulnerabilidad de salud y económica de los adultos mayores en México antes de la COVID-19. Novedades en Población, 17(33), 61-84.

Panorama de Envejecimiento | IADB. (s. f.). Recuperado 19 de julio de 2021, de https:// www.iadb.org/es/panorama/panorama-de-envejecimiento

Pinazo-Hernandis, S. (2020). Impacto psicosocial de la COVID-19 en las personas mayores: Problemas y retos. Revista Española de Geriatría y Gerontología, 55(5), 249-252. https://doi.org/10.1016/j.regg.2020.05.006

Price, B. (2015). Approaches to counter loneliness and social isolation. Nursing Older People, 27(7), 31-39. https://doi.org/10.7748/nop.27.7.31.e722

Quadagno, J. (2017). Aging and the Life Course: An Introduction to Social Gerontology. https://www.mheducation.com/highered/product/aging-life-course-introduction-social-gerontology-quadagno/M9781259870446.html

¿Quién cuida a los adultos mayores en América Latina y el Caribe? (2020, julio 7). Gente Saludable. https://blogs.iadb.org/salud/es/adultos-mayores-americalatina-caribe/

Ramos Bonilla, G. y Tirado Ratto, E. (2019). «Hasta que el cuerpo aguante». Trayectorias, rutinas y motivaciones laborales de trabajadores adultos mayores de la ciudad de Lima, Perú. Bulletin de l'Institut français d'études andines, 48(3), 381-404. https://doi.org/10.4000/bifea.11078

Ramos, M. Á. (s. f.). Contribución económica de las personas adultas mayores a los hogares urbanos en situación de pobreza en el Perú (p. 90).

Salgado Lévano, C. A. (2007). Investigación cualitativa: Diseños, evaluación del rigor metodológico y retos. Liberabit, 13(13), 71-78.

Sánchez-García, S., García-Peña, C., Salvà, A., Sánchez-Arenas, R., Granados-García, V., Cuadros-Moreno, J., Velázquez-Olmedo, L. B. y Cárdenas-Bahena, Á. (2017). Frailty in community-dwelling older adults: Association with adverse outcomes. Clinical Interventions in Aging, 12, 1003-1011. https://doi.org/10.2147/CIA. S139860

Seaman, A. T., Robbins, J. C. y Buch, E. D. (2019). Beyond the evaluative lens: Contextual unpredictabilities of care. Journal of Aging Studies, 51, 100799. https://doi. org/10.1016/j.jaging.2019.100799

Serrano-Martínez, C. (2020). Impacto emocional y crianza de menores de cuatro años durante el COVID-19. Perifèria. Revista d'investigació i formació en Antropologia, 25(2), 74-87. https://doi.org/10.5565/rev/periferia.735

Sureims, C. F., Omara Margarita, G. O., Virgen, C. S. y Ada María, de A. F. (2021, junio 28). Manifestaciones psicológicas en adultos mayores en aislamiento 
social durante la pandemia COVID-19. cibamanz2021. cibamanz2021. Recuperado de https://cibamanz2021.sld.cu/index.php/cibamanz/cibamanz2021/paper/ view/139

Victor, C. R., Scambler, S. J., Bowling, A. y Bond, J. (2005). The prevalence of, and risk factors for, loneliness in later life: A survey of older people in Great Britain. Ageing \& Society, 25(6), 357-375. https://doi.org/10.1017/S0144686X04003332

Villalba, C. G. Z. (2021). Revisión sistemática: Violencia intrafamiliar en tiempos de confinamiento por COVID 19. Perspectivas Metodológicas, 21, 14-14. https:// doi.org/10.18294/pm.2021.3605 